\title{
Development and Validation of UPLC Method for the Determination of Olopatadine Hydrochloride in Polymeric Nanoparticles
}

\author{
Umay Merve Güven ${ }^{1 *}$, Murat Sami Berkman², Yasemin Yazan² \\ 1 Cukurova University, Faculty of Pharmacy, Pharmaceutical Technology, Adana, Turkey. \\ 2 Anadolu University, Faculty of Pharmacy, Pharmaceutical Technology, Eskişehir, Turkey.
}

\begin{abstract}
A new, simple, rapid, precise, accurate and specific stability indicating reverse phase UPLC method developed for the determination of encapsulated olopatadine hydrochloride (OLO) in the polymeric nanoparticle formulations. Studies were carried out on a 2.1x50 mm, $1.8 \mu \mathrm{m}$ Zorbax Eclipse Plus $\mathrm{C}_{18}$ column with an optimized mobile phase of methanol, water and sodium acetate buffer solution (40:50:10, $\mathrm{v} / \mathrm{v} / \mathrm{v}$ ) at a flow rate of $0.5 \mathrm{~mL} / \mathrm{min}$. OLO was detected and quantitated using a photodiode array detector at a wavelength of $246 \mathrm{~nm}$ and the column temperature was adjusted to $40{ }^{\circ} \mathrm{C}$. The proposed method validation was carried out for specificity, linearity, accuracy, precision, limit of detection, limit of quantitation and robustness according to the ICH harmonised tripartite guideline "validation of analytical procedures Q2(R1)". Analytical curve was linear over the concentration range of $5-50 \mu \mathrm{g} / \mathrm{mL}$. All the validation parameters were within the acceptance range. LOD and LOQ for OLO were 0.7652 and $2.3188 \mu \mathrm{g} / \mathrm{mL}$, respectively. The developed method fulfilled the requirements for reliability and feasibility for the quantitative analysis of OLO in polymeric nanoparticles.
\end{abstract}

Keywords: Olopatadine hydrocloride, Eudragit, UPLC, validation, nanoparticles.

\section{INTRODUCTION}

The conventional eye drops are the most convenient and patient compliant non-invasive route for topical ophthalmic drug delivery. Nasolachrymal drainage, epithelial membrane barriers and non-productive absorption of these ophthalmic preparations can result in poor ocular bioavailability and systemic absorption leading side effects. The limited duration time requires frequently dosing up to 4 times per day for many treatments ${ }^{1}$. The active ingredient can

*Corresponding Author: Umay Merve Güven, e-mail: uguven@cu.edu.tr (Received 13 August 2018, accepted 20 September 2018) 
be delivered to the posterior ocular tissue segments by different administrations routes such as intravitreal injections, periocular injections, and systemic administration, but all have limitations. To overcome the ocular drug delivery barriers and improve bioavailability, various conventional and novel drug delivery systems have been developed such as emulsion, ointments, suspensions, aqueous gels, nanomicelles, nanoparticles, liposomes, dendrimers, implants, contact lenses, nanosuspensions, microneedles and in situ thermosensitive gels ${ }^{2,3}$.

Nanotechnology based ocular drug delivery systems such as polymeric nanoparticles have revealed promising results for dose optimization, bioavailability and sustained ocular drug delivery to the posterior ocular tissue segments.

Ultra-performance liquid chromatography (UPLC) is a new category of separation technique based upon well-established principles of liquid chromatograph $^{4,5}$. UPLC provides the benefit of small injection volumes, shortened run times ( $<5 \mathrm{~min}$ ), and reduced solvent usage, making it the more economical method for quantitation ${ }^{6}$. Because of its speed and sensitivity, this technique is gaining considerable attention in recent years for pharmaceutical and biomedical analysis ${ }^{7,8}$. In this study, UPLC was preferred for determination and quantification.

OLO (Figure 1) with histamine $\mathrm{H} 1$ receptor antagonistic action is used in ocular allergy and available on the market as eye drops for many years. OLO inhibits both mast cell degranulation and the release of arachidonic acid metabolites in various types of cells ${ }^{9,10,11}$. Therefore, OLO loaded polymeric nanoparticles were prepared as an alternative carrier to achieve the mentioned problems. The main purpose of this study was to describe a new assay for the determination of encapsulated OLO in the polymeric nanoparticle formulations.

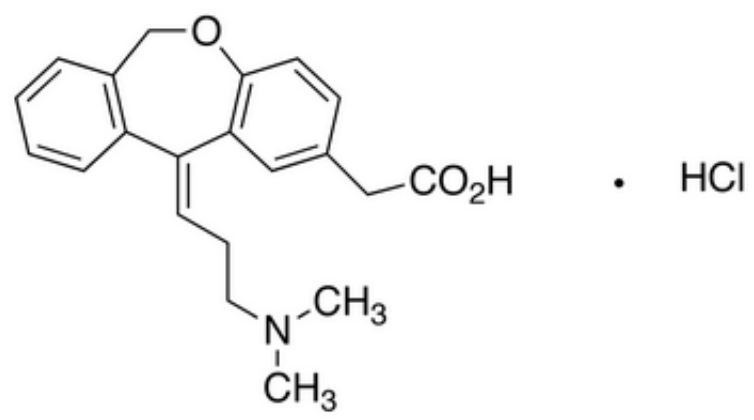

Figure 1. Olopatadine hydrochloride 


\section{METHODOLOGY}

\section{Materials}

Olopatadine hydrocloride, methanol and sodium acetate were purchased from Sigma (Steinheim, Germany). Eudragit ${ }^{\circledR}$ RS 100 was obtained from Degussa Röhm Pharma Polymers (Germany). All other reagents and solvents were of analytical grade and purchased from Sigma (Steinheim, Germany). High purity water was used throughout the experiment and prepared using a Millipore Milli-Q water (France) purification system.

\section{Preparation of Polymeric Nanoparticles}

Eudragit ${ }^{\circledR}$ RS 100 polymeric nanoparticles were prepared using spray-drying method $^{1}$. Briefly, Eudragit ${ }^{\circledR}$ RS 100 and OLO were dissolved in 100 mL methanol and stirred for 12 hours at room temperature with a magnetic stirrer. Final transparent solution was then spray-dried using a Mini Spray Dryer (B-190, BUCHI Labortechnik AG, Flawil, Switzerland) with an inlet and outlet temperature of $120{ }^{\circ} \mathrm{C}$ and $70{ }^{\circ} \mathrm{C}$, respectively. A white dry powder was obtained and kept in coloured vials at room temperature until analysis ${ }^{12,13}$. Olopatadin hydrocloride-free nanoparticles were also prepared as described above. Compositions of the nanoparticles prepared were given in Table 1.

Table 1. Compositions of the polymeric nanoparticles

\begin{tabular}{lccc}
\hline Code & OLO & Eudragit $^{\circledast}$ RS $\mathbf{~ 1 0 0 ~}(\mathbf{m g})$ & Methanol \\
& $(\mathbf{m g})$ & 500 & $(\mathbf{m L})$ \\
\hline NP plasebo & - & 500 & 100 \\
NP1 & 50 & 500 & 100 \\
NP2 & 75 & & 100 \\
\hline
\end{tabular}

\section{Chromatographic Conditions}

As a model of combined pharmaceutical applications, the chromatography analyses of the polymeric nanoparticles were performed using an Agilent 1290 Infinity UPLC system (Germany) equipped with a solvent degasser, quaternary pump, autosampler, column oven and diode array detector. Agilent ChemStation software was used for operation control and data collection. The method was developed using a 2.1x50 mm, $1.8 \mu \mathrm{m}$ Zorbax Eclipse Plus $\mathrm{C}_{18}$ column with an isocratic mobile phase consisting methanol, water and buffer solution in different ratios. The column temperature was maintained at $40{ }^{\circ} \mathrm{C}$ and UV detection was performed at $246 \mathrm{~nm}$. All prepared solutions were filtered through 
$0.22 \mu \mathrm{m}$ membrane filters before injection. Statistics were computed using Microsoft Excel 2010 (Redmond, WA, USA).

\section{Analytical Method Validation}

The UPLC method was validated according to the International Conference Harmonization ( $\mathrm{ICH}$ ) guideline ${ }^{2}$ with respect to specificity, linearity, accuracy and precision, limit of detection (LOD), limit of quantification (LOQ) and statistically evaluated for OLO quantification ${ }^{14}$.

\section{Linearity}

A linear relationship was evaluated across the range of the analytical procedure. The linearity of peak normalization (PN) ratios versus concentrations were evaluated using the calibration curve obtained from $5,10,15,20,25,30$, $35,40,45,50 \mu \mathrm{g} / \mathrm{mL}$ OLO solutions. Six individual replicates at each concentration were analyzed and $\mathrm{PN}$ ratios were calculated using the Equation 1.

$\mathrm{PN}=$ peak area / retention time $\left(\mathrm{R}_{\mathrm{t}}\right)$ Equation 1 .

Linear least squares methodology was used for the calculation of regression line. The correlation coefficient ( $\mathrm{r}$ ), coefficient of determination $\left(\mathrm{r}^{2}\right)$, y-intercept, slope of the regression line, residual sum of squares (RSS) was submitted with a plot of the data.

\section{Accuracy}

The accuracy of the method was determined by recovery studies evaluated in 3 concentration levels of 10,30 and $50 \mu \mathrm{g} / \mathrm{mL}$. Six individual replicates of each concentration were analyzed. Theoretical known amount and calculated assay amount were evaluated with the percent recovery.

\section{Precision}

The analyses were performed on the same day to determine repeatability or intra-day variability and on different days to establish the intermediate precision or inter-day variability. Samples were prepared in 3 concentration levels of $10,25,50 \mu \mathrm{g} / \mathrm{mL}$. Six individual replicate of each concentration were analyzed. The precision of the chromatographic method was reported as mean values, standart deviations (SD) and relative standart deviations (RSD) with confidence intervals.

\section{Specificity}

The specificity of the method was verified by comparing the chromatograms obtained from the placebo and OLO samples to show the detection ability of 
the desired components. Absence of any interference between the measured peaks was demonstrated in the representative chromatograms.

\section{Limit of Detection (LOD) and Limit of Quantification (LOQ)}

The calibration curve obtained in linearity was used for the detection limits. The LOD and LOQ were theoretically calculated by Equation 2 and 3 .

\section{$\mathrm{LOD}=3 \cdot 3 \sigma / \mathrm{S}$ Equation 2. \\ $\mathrm{LOQ}=10 \sigma / \mathrm{S}$ Equation 3 .}

where $\sigma$ is the standard deviation of $y$-axis interception values of the calibration curve and $\mathrm{S}$ is is the slope of the calibration curve ${ }^{15}$.

\section{Robustness}

The capacity of the analytical method was investigated by deliberate variation of the mobile phase composition, flow rate, column temperature and stability of OLO. Sample solutions were evaluated for each variation of the method conditions.

\section{Determination of OLO in Polymeric Nanoparticles}

Two different extraction methods were used to assess the encapsulation efficiency of the nanoparticles. $5 \mathrm{mg}$ nanoparticle was weighed and dispersed in $2 \mathrm{~mL}$ distilled water for the determination of superficial and free OLO. This suspension was centrifuged at $5000 \mathrm{rpm}$ for 3 minutes and filtered. The same amount of nanoparticles were dissolved in $2 \mathrm{~mL}$ methanol for the determination of total OLO. The mixture was vortexed for 1 minute and filtered. The loaded amount of OLO was determined by UPLC analyses of these two samples in triplicate. The measurements were also repeated at $3^{\text {rd }}$ and $6^{\text {th }}$ months for stability study. Encapsulation efficiency were calculated using the following Equation $4{ }^{16}$.

$\mathrm{EE}(\%)=\left[\left(\mathrm{OLO}_{\mathrm{T}}-\mathrm{OLO}_{\mathrm{S}}\right) \times\left(\mathrm{OLO}_{\mathrm{T}}\right)^{-1}\right] \times 100$ Equation 4 .

EE: Encapsulation Efficiency

$\mathrm{OLO}_{\mathrm{T}}$ : Total OLO content

$\mathrm{OLO}_{\mathrm{S}}$ : Superficial and free OLO content

\section{RESULTS AND DISCUSSION}

\section{Chromatographic Conditions}

Initial runs were carried out on methanol and water in various proportions to determime the appropriate mobile phase. Irregular shaping and tailing of OLO 
peaks were observed in isocratic mode. Satisfactory regular and symmetrical peaks were obtained by using 0.1 M sodium acetate buffer solution. Studies were carried out on a 2.1x50 mm, $1.8 \mu \mathrm{m}$ Zorbax Eclipse Plus C18 column with an optimized mobile phase of methanol, water and sodium acetate buffer solution (40:50:10, v/v/v) and the sample injection volume was $0.5 \mu \mathrm{L}$.

Independent variables such as oven temperature, flow rate and wavelength were also optimized which could greatly influence the separation procedure. The effect of oven temperature was studied between 20 and $40{ }^{\circ} \mathrm{C}$. The highest OLO peak area and shape/base compliance was achieved at an oven temperature of $40{ }^{\circ} \mathrm{C}$. The UV spectrum of OLO was scanned in the range of 200-400 $\mathrm{nm}$ and maximum absorption to accomplish the detection and the quantification of OLO was observed at $246 \mathrm{~nm}$. The role of the flow rate on retention time, elution and peak morphology was tested and mobile phase was found to be most reliable at flow rate of $0.5 \mathrm{~mL} \cdot \mathrm{min}^{-1}$. Under these conditions, OLO showed an acceptable retention time of $1.5 \mathrm{~min}$ with a run time of $5 \mathrm{~min}$. Analysis time was $7 \mathrm{~min}$ including the re-equilibration time.

Before analysis, the chromatographic column was equilibrated with the mobile phase for 30 minutes prior to injection. A summary of the chromatographic setup conditions for validation and analysis are represented in Table 2.

Table 2. Chromatographic conditions

\begin{tabular}{ll}
\hline stationary phase & Zorbax Eclipse Plus $\mathrm{C}_{18} 2.1 \times 50 \mathrm{~mm}, 1.8 \mu \mathrm{m}$ \\
mobile phase & Methanol: water:sodium acetate buffer solution (40:50:10, v/v/v) \\
oven temperature & $40^{\circ} \mathrm{C}$ \\
flow rate & $0.5 \mathrm{~mL} / \mathrm{min}$ \\
injection volume & $0.5 \mu \mathrm{L}$ \\
detection wavelenght & $246 \mathrm{~nm}$ \\
\hline
\end{tabular}




\section{Analytical Method Validation}

Calibration curves were obtained by plotting the PN ratios versus concentrations after the analysis of the injected samples (Figure 2).

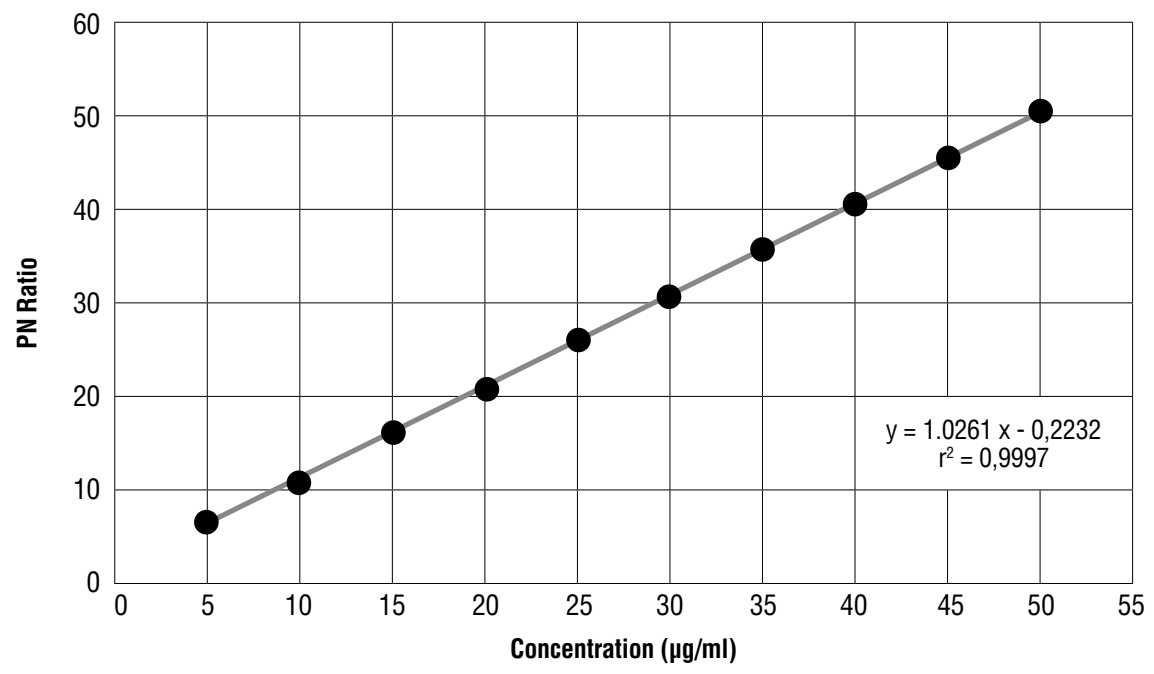

Figure 2. Linearity equation of OLO.

The linearity of the method was established in the $5-50 \mu \mathrm{g} / \mathrm{mL}$ OLO range and showed excellent correlation within the concentration range. Regression statistics data of the six individual replicates were summarized in Table 3.

Table 3. Regression statistics of linearity $(n=6)$

\begin{tabular}{lcc}
\hline & pooled & mean \\
\hline correlation coefficient $(r)$ & 0.9984 & 0.9999 \\
coefficient of determination $\left(\mathrm{r}^{2}\right)$ & 0.9968 & 0.9997 \\
observations & 60 & 10 \\
y-intercept & & -0.2232 \\
slope & \multicolumn{2}{c}{1.0261} \\
\hline
\end{tabular}

The coefficient of determination close to unity was not the necessarily outcome of a linear relationship and the use of only this value could be potentially misleading according to the Analytical Methods Committee (AMC) technical brief ${ }^{17}$. Therefore, the lack-of-fit test was applied as an auxiliary indicator for linearity by evaluating the variance of the residual values (Table 4). Analysis of Vari- 
ance (ANOVA) results were calculated by Minitab ${ }^{\circledR} 18$ data analysis sofware and confirmed linearity significance of the curve, homogeneity of variances, and normality of the residues. The lack-of-fit was not statistically significant as $P$-value $>0.05$ (0.754) which means the test did not detect any lack-of-fit at the a level in the simple linear regression model.

Table 4. ANOVA linearity pooled results ( $n=6)$ (DF: degrees of freedom; SS: sum of squares; MS: mean squares; F: F-value; $P$ : $P$-value)

\begin{tabular}{lccccc}
\hline & DF & SS & MS & $\mathbf{F}$ & $\mathbf{P}$ \\
\hline regression & 1 & 13028.4 & 13028.4 & 17900.21 & 0.000 \\
residual & 58 & 42.2 & 0.7 & & \\
$\quad$ lack-of-fit & 8 & 3.8 & 0.5 & 0.62 & 0.754 \\
$\quad$ pure error & 50 & 38.4 & 0.8 & & \\
total & 59 & 13070.7 & & & \\
\hline
\end{tabular}

The accuracy of the method was determined by recovery studies of the known contentrations. Analysis was carried out by the proposed method. The percentage recovery data were found to be accurate and in the acceptance limit of $\pm 2 \%$. The results indicated a low variability and a strong agreement between the theoretical known amount and calculated assay amount. The percentage recovery data, difference between mean and accepted true values and confidence intervals were presented in Table 5 .

Table 5. Accuracy results $(n=6)$

\begin{tabular}{lccc}
\hline concentration levels $(\mu \mathrm{g} / \mathrm{mL})$ & 10 & 30 & 50 \\
\hline recovery $\%$ & 100.8911 & 99.8584 & 100.4588 \\
difference $(\mu \mathrm{g} / \mathrm{mL})$ & 0.0891 & 0.0425 & 0.2294 \\
confidence intervals $(95 \%)$ & 0.1354 & 0.2918 & 0.5817 \\
\hline
\end{tabular}

The precision study was performed on three concentration levels (low, medium, and high) to evaluate the repeatability and intermediate precision of the analytical method. Analyses were carried out on three consecutive days to show the intra-day and inter-day variations. The precision of the method was verified and found to be within the targeted intervals since the RSD is below $2 \%$. The precision data were presented in Table 6 . The inter-day results of the three days were pooled and analyzed by GraphPad Prism 7 software. 
Table 6. Intra-day and inter-day (pool-days) precision results $(n=6)$

\begin{tabular}{|c|c|c|c|c|c|}
\hline \multirow{2}{*}{$\begin{array}{l}\text { concentration } \\
\text { levels }(\mu \mathrm{g} / \mathrm{mL})\end{array}$} & & \multicolumn{3}{|c|}{ intra-day } & \multirow{2}{*}{ inter-day } \\
\hline & & day 1 & day 2 & day 3 & \\
\hline \multirow{4}{*}{10 (low) } & Mean & 10.5235 & 10.0891 & 10.0709 & 10.2278 \\
\hline & SD & 0.1965 & 0.1290 & 0.1906 & 0.2707 \\
\hline & RSD & 1.8675 & 1.2786 & 1.8921 & 0.0277 \\
\hline & $\mathrm{Cl}(95 \%)$ & 0.2062 & 0.1354 & 0.2000 & 0.1346 \\
\hline \multirow{4}{*}{25 (medium) } & Mean & 25.5769 & 25.6812 & 25.8976 & 25.7186 \\
\hline & SD & 0.3612 & 0.4332 & 0.4008 & 0.3996 \\
\hline & RSD & 1.4120 & 1.6867 & 1.5478 & 1.5538 \\
\hline & $\mathrm{Cl}(95 \%)$ & 0.3790 & 0.4546 & 0.4206 & 0.1987 \\
\hline \multirow{4}{*}{50 (high) } & Mean & 49.7572 & 50.4572 & 50.2294 & 50.1479 \\
\hline & SD & 0.7992 & 0.7875 & 0.5543 & 0.7420 \\
\hline & RSD & 1.6063 & 1.5607 & 1.1036 & 1.4797 \\
\hline & $\mathrm{Cl}(95 \%)$ & 0.8387 & 0.8264 & 0.5817 & 0.3690 \\
\hline
\end{tabular}

The specificity of the developed method was conducted with OLO loaded and placebo formulations. It was determined that overlapping effect of other formulation components did not affect the OLO peak (Figure 3). It was therefore concluded that the developed method was specific and the OLO peak was distinctly separated from other components in the formulations.

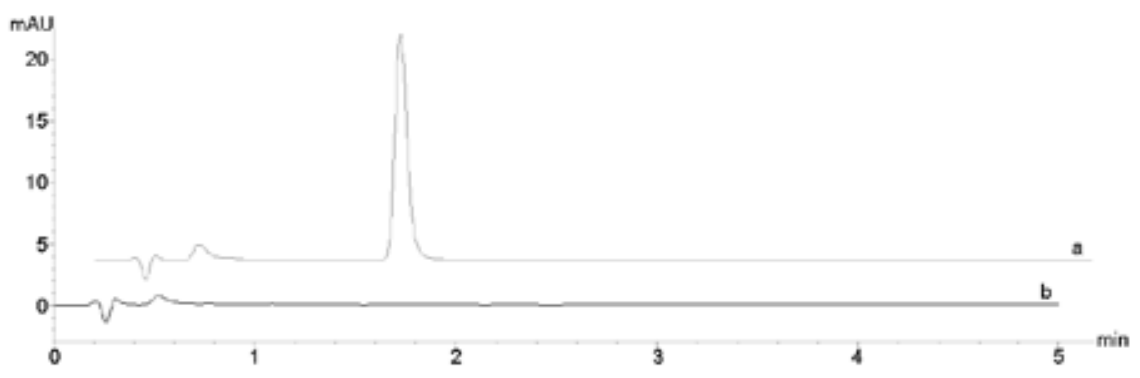

Figure 3. Chromotogram of OLO (a) and placebo formulations (b)

The parameters LOD and LOQ were calculated using the standard deviation of y-axis interception values of the calibration curve and the slope of the calibration curve. The lowest OLO concentration detected and quantified were 0.7652 and $2.3188 \mu \mathrm{g} / \mathrm{mL}$ respectively. Calculated LOD and LOQ concentrations could be considered as relatively high. The possible reasons of this situation were the use of PDA detector or high content of organic solvent and partial UV-cutoff effect. Nevertheless, these results proved that the chromatographic 
method was suitable enough to detect and quantify OLO at a concentration range of 5 to $50.0 \mu \mathrm{g} / \mathrm{mL}$.

The robustness of the developed method was investigated with slight changes in the column temperature, $\mathrm{pH}$ of the mobile phase and flow rate. However, these changes had an influence on the assay and stability, the method was considered robust as the RSD values were below $2 \%$ for the OLO content.

\section{Determination of OLO in Polymeric Nanoparticles}

The OLO content and encapsulation efficiency of the polymeric nanoparticles was carried out by the validated UPLC method. The results of characterizations and 6 months stability of the formulations $\left(25^{\circ} \mathrm{C}\right.$ and $\left.60 \% \mathrm{RH}\right)$ were presented in Table 7. The entrapment efficiency was evaluated according to Equation 4. Superficial and free OLO content was found higher than the encapsulated for both NP1 and NP2 formulations. Drug and polymer concentration ratios was found to be a significant factor for the entrapment efficiency ${ }^{18}$. Although the amount of OLO in the NP2 formulation was greater than NP1, NP2 was less loaded. As a result of the stability studies over 6 months, no statistically significant change was observed ( $\mathrm{p}>0.05$ ) in $\mathrm{OLO}_{\mathrm{T}}$, OLOS and the entrapment efficiency.

Table 7. The entrapment efficiency of the polymeric nanoparticles

\begin{tabular}{lcccc}
\hline time & code & $\mathbf{O L O}_{\mathbf{T}}(\boldsymbol{\mu g} \pm \mathbf{S E})$ & $\mathbf{O L O}_{\mathbf{S}}(\boldsymbol{\mu g} \pm \mathbf{S E})$ & $\mathrm{EE}(\%)$ \\
\hline initial & $\mathrm{NP1}$ & $140.007 \pm 0.738$ & $102.138 \pm 0.874$ & 27.048 \\
& $\mathrm{NP2}$ & $203.680 \pm 0.532$ & $184.990 \pm 0.587$ & 9.176 \\
3 months & $\mathrm{NP1}$ & $141.422 \pm 0.560$ & $103.436 \pm 0.755$ & 26.983 \\
& $\mathrm{NP2}$ & $208.602 \pm 0.797$ & $188.783 \pm 0.847$ & 9.530 \\
6 months & $\mathrm{NP1}$ & $144.755 \pm 1.172$ & $106.270 \pm 0.847$ & 26.706 \\
& $\mathrm{NP2}$ & $213.602 \pm 1.161$ & $192.783 \pm 0.524$ & 9.776 \\
\hline
\end{tabular}

\section{CONCLUSION}

Analytical method validation was performed to confirm that the analytical procedure developed was adequate for its intended use, and that the results derived could be utilized to determine the reliability and consistency of the analytical data obtained. The method was completely validated for linearity, accuracy, precision, specificity, LOD, LOQ and robustness according to the ICH harmonised tripartite guideline "validation of analytical procedures Q2(R1)" and showed satisfactory data for all tested parameters. As a result, this method particularly exhibited an excellent sensitivity and speed performance for the 
determination of OLO.

This newly developed UPLC method was also successfully applied for the determination of OLO in polymeric nanoparticle formulations, encapsulation efficiency and the stability studies. The results were found within higher confidence. In conclusion, this stability indicating method can be used and adaptable for the determination of OLO in similar pharmaceutical dosage forms.

\section{ACKNOWLEDGEMENTS}

This research was supported by Anadolu University Scientific Research Project Committee (No: 1406S312).

\section{CONFLICTS OF INTEREST}

The authors report no conflict of interest. The authors alone are responsible for the content and writing of the paper.

\section{REFERENCES}

1. González-Chomón, C.; Silva, M.; Concheiro, A.; Alvarez-Lorenzo, C. Biomimetic contact lenses eluting olopatadine for allergic conjunctivitis. Acta Biomaterialia. 2016, 41, 302-311.

2. Patel, A.; Cholkar, K.; Agrahari, V.; Mitra, A. K. Ocular drug delivery systems: An overview. World J Pharmacol. 2013, 2(2), 47-64.

3. Abelson, M. B.; Smith, L.; Chapin, M. Ocular allergic disease: mechanisms, disease subtypes, treatment. Ocul Surf. 2003, 1(3), 127-149.

4. Nováková, L., Matysová, L., \& Solich, P. (2006). Advantages of application of UPLC in pharmaceutical analysis. Talanta, 2006, 68(3), 908-918.

5. Dewani, A. P.; Dabhade, S. M.; Bakal, R. L.; Gadewar, C. K.; Chandewar, A. V.; Patra, S. Development and validation of a novel RP-HPLC method for simultaneous determination of paracetamol, phenylephrine hydrochloride, caffeine, cetirizine and nimesulide in tablet formulation. Arabian Journal of Chemistry, 2015, 8(4), 591-598.

6. Addo, R. T.; Davis, K.; Ubale, R.; Owen, J. S.; Watkins, E. B. Development and validation of a UPLC method for rapid and simultaneous analysis of proton pump inhibitors. AAPS PharmSciTech, 2015, 16(1), 30-34.

7. Johnson, K. A; Plumb, R. Investigating the human metabolism of acetaminophen using UPLC and exact mass oa-TOF MS. J Pharm Biomed Anal. 2oo5, 39(3-4), 805-810.

8. Kadav, A.A; Vora, D.N. Stability indicating UPLC method for simultaneous determination of atorvastatin, fenofibrate and their degradation products in tablets. $J$ Pharm Biomed Anal. 2008, 48(1), 120-126.

9. Reddy, Y.R; Kumar, K.K; Reddy. M.; Mukkanti, K. RP-UPLC method development and validation for the simultaneous estimation of ibuprofen and famotidine in pharmaceutical dosage form. Pharm Methods. 2012, 3(2), 57-61.

10. Matsubara, M.; Masaki, S.; Ohmori, K.; Karasawa, A. Hasegawa, K. Differential regulation of IL-4 expression and degranulation by anti-allergic olopatadine in rat basophilic leukemia 
(RBL-2H3) cells. Biochemical Pharmacology. 2o04, 67, 1315-1326.

11. Tamura, T.; Komai, M. Effect of olopatadine hydrochloride, an anti-histamine drug, on rhinitis induced by intranasal instillation of toluene-2,4-diisocyanate in rats. International Immunopharmacology. 2008, 8, 916-921.

12. Shukla, M. H.; Patel, A. P.; Patel, M. G.; Patel, D. P.; Shah, R. R. Development and validation of first order derivative spectroscopic method for estimation of olopatadine hydrochloride and ambroxol hydrochloride in their synthetic mixture. Pharma Science Monitor, 2015, $6(1)$.

13. Başaran, E. Ocular application of dirithromycin incorporated polymeric nanoparticles: An in vitro evaluation. Turk J Pharm Sci. 2017, 14(2), 191-200.

14. ICH Harmonized Tripartite. Validation of analytical procedures: Text and methodology. Guideline. 2005, Q2(R1).

15. Berkman, M. S.; Yazan, Y. A validated HPLC method for the determination of octocrylene in solid lipid nanoparticle systems. Pharmazie. 2011, 66, 105-110.

16. Vardhan, H.; Mittal, P.; Adena, S. K. R.; Mishra, B. Long-circulating polyhydroxybutyrateco-hydroxyvalerate nanoparticles for tumor targeted docetaxel delivery: Formulation, optimization and in vitro characterization. Eur J Pharm Sci. 2017, 99, 85-94.

17. Amc Technical Brief: Is my calibration linear? Analytical Methods Committee. 2005.

18. Wang, H.; George, G.; Bartlett, S.; Gao, C.; Islam, N. Nicotine hydrogen tartrate loaded chitosan nanoparticles: Formulation, characterization and in vitro delivery from dry powder inhaler formulation. Eur J Pharm Biopharm. 2017, 113, 118-131. 